\title{
AN OVERVIEW OF INTERFACE CRACKS
}

\author{
MARIA COMNINOU \\ Mechanical Engineering and Applied Mechanics, University of Michigan, Ann Arbor, \\ MI 48109-2125 U.S.A.
}

\begin{abstract}
In many cases cracks leading to fracture occur at interfaces between two different constituents, e.g. a fiber and the matrix in a composite. The early solutions of such problems in the context of linear elastic fracture mechanics (LEFM) revealed the presence of an unsatisfactory behavior: rapid oscillations in the stress and displacement fields, implying the physically impossible phenomenon of interpenetration. In the late seventies two major modifications were proposed: one by Atkinson and the other by Comninou. The Atkinson modification recognizes that the interface between two different materials is almost never sharp, and provides a gradual transition which avoids the oscillatory behavior. The Comninou approach simply resolves the interface crack problem by accepting its inherently unilateral nature (presence of inequalities) and allowing for partial closure at the tips. Both solutions have received high praise and severe criticism, especially since the oscillatory behavior is absent in some, at least, classes of nonlinear materials (Knowles and Sternberg). Current emphasis is placed on numerical models of the elastoplastic behavior of interface cracks. An additional complication is the apparent presence of mixed mode crack tip fields regardless of the type of the applied loading. Valuable as these theoretical efforts may be, it is becoming increasingly imperative to perform experiments to determine the mode of propagation and critical parameters governing interface fracture. Even then, the results must be viewed with caution, because the quantities of interest can only be determined indirectly. The present paper presents an overview of the interface crack problem and describes some preliminary experimental results in the fatigue and fracture of interface cracks.
\end{abstract}

\section{INTRODUCTION}

THERE HAS been a recent resurgence of interest on the subject of interface cracks, stemming mainly from the desire to understand the modes of failure of composites, including debonding and delamination. On the theoretical side, new efforts include the exploration of the regime of validity of LEFM solutions, the search for design parameters characterizing failure, and the development of elasto-plastic numerical solutions. On the experimental side, a lot of work is devoted to the study of the interface, its character and behavior, in materials science terms. The intriguing and paradoxical nature of the problem has divided investigators into several camps with no clear consensus emerging as yet. The sophistication of published work ranges from cases of sheer elegance to cases of borderline quackery; the literature has become a minefield for the neophyte.

The paper reviews some of the representative work of the past two decades on interface cracks.

\section{REVIEW: LEFM SOLUTIONS}

Theoretical investigations of the interface crack problem date since the late fifties. Williams[1] performed an asymptotic analysis of the elastic fields at the tip of a open interface crack and found that the stresses and displacements behaved in an oscillatory manner. Subsequent solutions retained this feature. Erdogan[2] considered the nonhomogeneous plane with cracks and found that the extent of the oscillatory region is of the order of $10^{-6}$ of the crack length. Rice and Sih[3] obtained a Green's function solution for the finite interface crack. England[4] studied the interface crack under arbitrary internal pressure, and noted that the solution is physically inadmissible "since it predicts that the upper and lower surfaces of the crack should wrinkle and overlap near the ends of the crack". Using the oscillatory solution, England computed the distance from the crack tip that corresponds to the first interpenetration. This distance, found to be of the order of $10^{-4}$, gives an estimate of the extent of the contact zone. Malyshev and Salganik[5] also discussed the implications of the oscillatory fields and made the following comment: "For opposite faces of the cut the result is physically absurd, that is they are penetrating each other. The fault of the mathematical model can be corrected if it is supposed that the opposite faces taking mutually convex shape start to press in each other forming contacting areas." They also argued that if the 
length of the cohesion zone in a Barenblatt-Dugdale type of model is greater than the region of stress oscillations, then the latter can be disregarded near the crack tip.

There are other drawbacks associated with the oscillatory behavior of the elastic fields. The problem ceases to be self-similar, and the absence of a characteristic length in the asymptotic analysis poses a dimensional question. Modes I and II are coupled, and it is not clear what is the proper definition of the stress intensity factors. From Williams asymptotic analysis we see that the stresses and displacements behave as

$$
\begin{aligned}
& \sigma \sim r^{-1 / 2}(\sin , \cos )(\epsilon \log r) \\
& u \sim r^{1 / 2}(\sin , \cos )(\epsilon \log r) \\
& \epsilon=\frac{1}{2 \pi} \log \left(\frac{1-\beta}{1+\beta}\right)
\end{aligned}
$$

where $\beta$ is a dimensionless composite parameter depending on the material properties and introduced by Dundurs[6]. It varies from $-1 / 2$ to $1 / 2$ and vanishes for identical materials. It also vanishes for two incompressible materials, or one incompressible and the other rigid under conditions of plane strain, among other combinations:

$$
\beta=\frac{\mu_{2}\left(\kappa_{1}-1\right)-\mu_{1}\left(\kappa_{2}-1\right)}{\mu_{2}\left(\kappa_{1}+1\right)+\mu_{1}\left(\kappa_{2}+1\right)}
$$

where $\kappa=3-4 v$ for plane strain and $\kappa=(3-4 v) /(1+v)$ for plane stress, and $\mu, v$ are the shear modulus and Poisson's ratio respectively. In plane strain $\beta$ is a measure for the mismatch in bulk moduli.

From eq. (1) it is seen that the usual definition of the stress intensity factors as $\lim _{r \rightarrow 0}\left\{(2 \pi r)^{1 / 2} \sigma\right\}$ will not work: it produces logarithmically infinite factors. Furthermore, any attempt to define the stress intensity factors without reference to a characteristic length, such as the crack length, will produce dimensionally meaningless results. However, the strain energy release rate $G$ computed by the dimensionally wrong factors will still be dimensionally valid. The stress intensity factors $k_{1}$ and $k_{2}$ for an interface crack of length $2 a$ in a uniformly applied tension field $T$ as defined by Rice and Sih[3] contain the logarithm of $2 a$ :

$$
\begin{aligned}
k= & k_{1}+i k_{2}=T(\pi a)^{1 / 2}\{[\cos (\epsilon \log 2 a)+2 \epsilon \sin (\epsilon \log 2 a)] \\
& -i[\sin (c \log 2 a)+2 c \cos (c \log 2 a)]\} / \cosh \pi c .
\end{aligned}
$$

However, the expression $\left(k_{1}^{2}+k_{2}^{2}\right)$, which is proportional to $G$, does not contain logarithms:

$$
k_{1}^{2}+k_{2}^{2}=T^{2} \pi a\left(1+4 \epsilon^{2}\right) / \cosh ^{2} \pi \epsilon
$$

For the same problem, Frdogan and Gupta[7] used the following definition:

$K_{1}+i K_{2}=(\pi / a)^{1 / 2} \lim _{x \rightarrow a}(x-a)^{-1 / 2-i c}(x+a)^{1 / 2+i c}\left(\sigma_{y y}+i \sigma_{x y}\right)$

and obtained:

$$
K_{1}+i K_{2}=T(\pi a)^{1 / 2}(1+2 i \epsilon)
$$

Recently, Rice[8] and Hutchinson et al.[9] have been advocating a complex stress intensity factor $K^{*}$ defined as follows

$$
\sigma_{y y}+i \sigma_{x y}=K^{*}(2 \pi r)^{-1 / 2} r^{i} ; \quad K^{*}=K_{1}^{*}+i K_{2}^{*}
$$

$K^{*}$ is simply a new version of $k$ :

$$
K^{*}=k \cosh \pi \epsilon
$$

and it contradicts general ideas about units and dimensional consistency. Rice suggests the use of the unit MPam ${ }^{1 / 2} \mathrm{~m}^{-i c}$, which again implies taking the logarithm of a length. Although he also introduces an arbitrary scaling length as an alternative, there seems to be no way to reconcile the difficulties. 


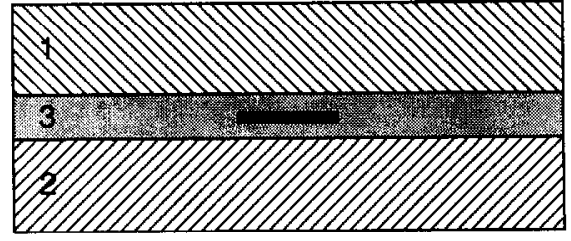

Fig. 1. Atkinson's first model: homogeneous interface layer with crack.

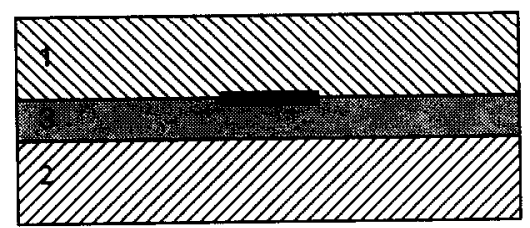

Fig. 2. Atkinson's second model: interface layer with continuously varying moduli.

Various attempts have been made to resolve the difficulties associated with the oscillatory singularities. In the context of LEFM the modifications proposed by Atkinson[10] and Comninou[11] have received attention in the form of praise, criticism and follow-up work. Atkinson proposed two models. In both models the interface is replaced by a thin strip of finite thickness. In the first model, Fig. 1, the strip is homogeneous and has elastic moduli which are different than the moduli of the other two solids. The crack is placed inside the homogeneous layer, and therefore oscillatory singularities do not occur. This model has the shortcomings that there are still two discontinuous interfaces and that the crack is constrained to lie in the interior of the interface layer. For this reason it has not gained much popularity. In the second model, Fig. 2, the interface layer has continuously varying moduli, and equal to those of the surrounding solids at its boundaries. The crack is placed along the interface between the first solid and the layer, but, because the moduli are continuous, there is no oscillatory behavior and the singularity at the crack tip is of the usual square-root type. This model is more realistic, especially for combinations of materials and processes producing a diffuse interface, and interest in it has revived recently. The Comninou or contact zone model, Fig. 3, is not a model at all. It follows the suggestion of Malyshev and Salganik[5] allowing the crack faces to come in to contact near the crack tips. The extent of contact is unknown, and must be determined as part of the solution. The formulation recognizes the unilateral nature of the crack problem and provides the only valid solution of the true interface crack in the context of LEFM. The existence and uniqueness of the solution follow from the treatment of the Signorini problem, (Fichera[12]). Shield[13] has also proved uniqueness specifically for the interface crack which is partially open. However, since LEFM may or may not be appropriate for the description of propagation characteristics of interface cracks, the contact zone solution may or may not be useful, regardless of its mathematical correctness.

The asymptotic behavior of the closed crack tip, Comninou[11], is unfamiliar and possibly disconcerting, Fig. 4. Ahead of the crack tip, that is on the bond side, only the shear tractions are singular, exhibiting the square-root type of singularity, regardless of the nature of the applied loads. The normal tractions are not singular, although they may be large. The compressive tractions in the contact zone have a square-root singularity behind the crack tip (on the side of the contact). We may define a stress intensity factor $K_{1}^{-}$associated with compression, which must then be negative. There is a relation between $K_{\mathrm{I}}^{-}$and $K_{\mathrm{ll}}$ :

$$
K_{\mathrm{I}}^{-}= \pm \beta K_{\mathrm{II}}
$$

where the + sign applies when the bond is to the left of the crack tip, and the - sign when the bond is to the right of the crack tip. Since $K_{\mathrm{I}}^{-}$is always negative, it follows that $K_{\mathrm{II}}$ has opposite

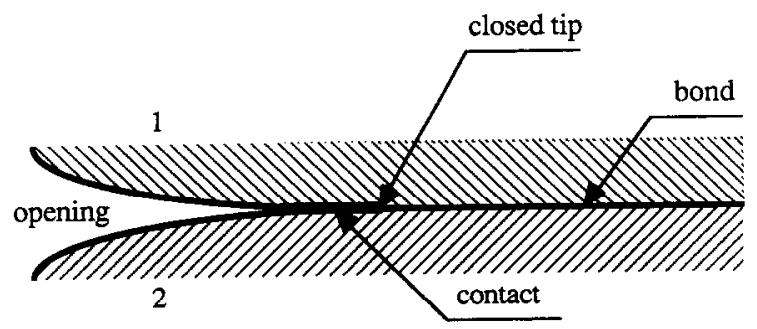

Fig. 3. Contact zone model. 


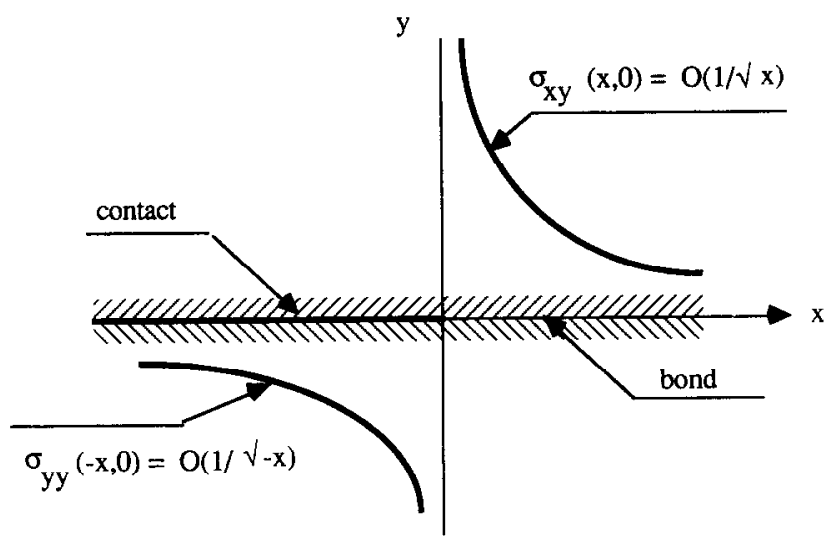

Fig. 4. Asymptotics of the closed crack tip.

signs at the two tips of a finite crack. It is important to keep this observation in mind when performing numerical calculations in which the sign of the contact tractions near the crack tip cannot be determined accurately. Accordingly, the finite element calculations of van der Zande and Grootenboer[14] leading to positive values of $K_{\text {II }}$ at both crack tips of a finite crack in shear, cannot be correct (please note that only their algebraic signs are suspect, and not the entire computations). Although the contact zone formulation has a unique correct solution, it has also an infinity of wrong solutions corresponding to contact zones of smaller lengths and interpenetration, Atkinson[15]. Therefore, it is not sufficient to monitor the contact stresses alone; the crack opening displacement must also be shown to be positive.

The preceding discussion gives an indication of the subtleties involved in obtaining the correct solution of a given formulation for the interface crack. Regarding the contact zone model, Comninou's solution, based on the numerical quadrature of a singular integral equation with nonlinear dependence on the unknown contact length, was only accurate for a small range of the parameter $\beta$. Recent results by Gautesen and Dundurs $[16,17]$ must be consulted for accurate calculations in the entire range of $\beta$. Even for the usual interface crack formulation (open crack tips and oscillatory singularities), there are authors who claim to have found oscillation-free solutions, Itou[18], Zhang[19].

The interface crack in tension. For the interface crack in tension the contact zone model provides a contact zone of the order of $10^{-4}$ of the crack length (Comninou[11], Gautesen and Dundurs[16]) for $\beta=0.5$, diminishing to zero as $\beta$ approaches zero. On the other hand, an estimate of the extent of the contact zone can be obtained from the oscillatory solution computing the distance $\delta$ from the crack tip at which the crack opening displacement first becomes zero (implying contact). The expression for $\delta$ normalized with the crack length $2 a$ is given by England[4] as:

$$
\frac{\delta}{2 a}=\frac{1}{1+\mathrm{e}^{\pi / \omega}}
$$

The same quantity is computed from the contact zone model from the expression given by Gautesen and Dundurs [16] with accuracy to four significant figures:

$$
\begin{aligned}
\frac{\delta}{2 a} & =\frac{1}{2}\left[1-\left(1-\lambda^{2}\right)^{1 / 2}\right] \\
\lambda^{2} & =16 \exp \left\{\left[2 \tan ^{-1}(\omega)+\pi\right] / \omega\right\}
\end{aligned}
$$

where $\omega=-2 \epsilon$ is taken as positive without loss of generality, since $\beta$ changes sign when the labeling of the solids is interchanged. The results are shown in Table 1. It is noted that the contact estimated from the overlap is 70 to $85 \%$ greater than the true contact. Estimated contacts from overlaps are usually larger than the actual contacts: the ratio of the overlap over the contact area of the axisymmetric Hertz problem is 2 . 
Table 1 .

\begin{tabular}{|c|c|c|c|}
\hline$\beta$ & $\begin{array}{c}\delta / 2 a \\
\text { England }\end{array}$ & $\begin{array}{c}\delta / 2 a \\
\text { Gautesen } \\
\text { Dundurs }\end{array}$ & ratio \\
\hline 0.05 & $1.488 \mathrm{E}-43$ & 8.060-E44 & 1.846 \\
\hline 0.1 & $4.366 \mathrm{E}-22$ & $2.370 \mathrm{E}-22$ & 1.842 \\
\hline 0.15 & $6.608 \mathrm{E}-15$ & 3.599E-15 & 1.836 \\
\hline 0.2 & $2.683 \mathrm{E}-11$ & $1.469 \mathrm{E}-11$ & 1.827 \\
\hline 0.25 & $4.065 \mathrm{E}-09$ & 2.239E-09 & 1.815 \\
\hline 0.3 & $1.191 \mathrm{E}-07$ & $6.612 \mathrm{E}-08$ & 1.801 \\
\hline 0.35 & $1.366 \mathrm{E}-06$ & 7.658E-07 & 1.784 \\
\hline 0.4 & $8.734 \mathrm{E}-06$ & 4.953E-06 & 1.763 \\
\hline 0.45 & $3.788 \mathrm{E}-05$ & 2.177E-05 & 1.739 \\
\hline 0.5 & $1.254 \mathrm{E}-04$ & $7.327 \mathrm{E}-05$ & 1.712 \\
\hline
\end{tabular}

Energy considerations. The strain energy release rate $G$, which is equal to the $J$ integral in LEFM, is obtained for the contact model as

$$
G^{c}=\left(c_{1}+c_{2}\right)\left(1-\beta^{2}\right)\left(K_{\mathrm{II}}^{c}\right)^{2} / 16,
$$

and for the oscillatory solution as

$$
G^{0}=\left(c_{1}+c_{2}\right) K^{*} \bar{K}^{*} / 16 \cosh ^{2} \pi \epsilon
$$

where $c_{i}=\left(1+\kappa_{i}\right) / \mu_{i}$. From very small contact zones, the fields far from the crack tip are not affected by the presence of contact. Fquating $G^{c}$ and $G^{0}$ gives an approximate expression for $K_{\mathrm{II}}^{c}$, Park and Earmme[20]. For interface cracks in uniform tension $T$ the result is

$$
K_{\mathrm{i}}^{c}=\left[\pi\left(1+4 \epsilon^{2}\right) a\right]^{1 / 2} T .
$$

The corresponding energy release rate was also obtained by Comninou[11] using a different argument.

The interface crack in shear. Under pure shear load the linear elasticity solution, which considers the crack completely traction-free and open, is antisymmetric. As a consequence, overlapping or negative gap is obtained over half the extent of the contact in addition to the oscillatory behavior. When the problem is solved under unilateral conditions, i.e. when contact between the crack faces is allowed at the tips, a finite contact zone with length of the order of a third of the crack length is obtained adjacent to one crack tip, while an infinitesimal zone is predicted next to the other tip, Comninou[21]. If the materials are interchanged or the direction of the applied shear is reversed, the contact zones exchange magnitude. It is also interesting to note that the mode II stress intensity factors also exchange magnitude, but retain their algebraic signs. This result is predicted by asymptotic analysis, Comninou[11], Comninou and Dundurs[22].

Combined loads. The effects of closure on the interface crack under combined shear and normal loads were first considered by Comninou and Schmueser[23], and recently by Gautesen and Dundurs[17], who obtained asymptotically exact results. They found that the extent of the large contact zone increases or decreases with $\beta$ depending on the algebraic sign of the ratio of the normal over the shear stress, but there is little variation with $\beta$ when this ratio is zero. The agreement between the two papers is quite good except for the mode II stress intensity factors of the tip adjacent to the small contact zone: those given by Comninou and Schmuser are inaccurate in magnitude. A finite element analysis by van der Zande and Grootenboer[14], on the other hand, predicts a change in the algebraic sign of the mode II stress intensity factor at the side of the small contact zone which is in conflict with the accurate results of Gautesen and Dundurs.

\section{NONLINEAR AND ELASTOPLASTIC CONSIDERATIONS}

It has been repeatedly asserted that the problems of the oscillatory singularities are a mere creation of the linear elasticity theory and, therefore, any attempts to provide corrections within this theory are invalid or unnecessary, since linear elasticity is inapplicable. Credence was given 
to this point of view by the work of Knowles and Sternberg[24]. Using an asymptotic investigation, they have shown that an interface crack between two incompressible neo-Hookean sheets in plane stress does not involve oscillatory singularities or material interpenetration requiring contact at the tips. In the absence of general results, it is just as possible to argue that the absence of oscillatory behavior in the nonlinear solution is due to the peculiarities of the material chosen. This argument is reinforced by the fact that, for the homogeneous case in mode II crack problems, nonlinear effects may lead to either crack-opening or material interpenetration, Knowles[25], in contrast to linear elasticity results. To quote Knowles, using experimentally determined first- and second- order elastic constants "it is found that crack-opening occurs for polystyrene and iron, but interpenetration is predicted for glass". Examples show that "crack-opening may occur despite the presence of a compressive normal stress $\tau_{y y}$ at infinity, and that interpenetration of the crack-faces can be predicted even though the normal stress is tensile", according to the same author. It seems then that either the nonlinear theory is rich enough to predict a variety of effects, or that the linear theory misrepresents the local behavior of cracks, whether they are at interfaces or not. As for the global solution based on linearized theories, Knowles and Sternberg state that "the precise approximative status of solutions to linearized problems involving interface-cracks remains an intriguing issue". It should also be noted that the actual stresses in the neo-Hookean solution exhibit singularities greater than $1 / 2$, a result which is consistent with the hardening character of these materials, Knowles and Sternberg[24].

More promising seems the outlook offered by elasto-plastic analyses of interface cracks. Such analyses are implemented by a numerical method, usually the infinite element method. Shih and Asaro[26] have obtained some results for small scale yielding at the crack tip. Representing the strains as a sum of a linear elastic term and a plastic term, their preliminary results appear to indicate the existence or extent of contact depends on whether elastic or incompressibility effects dominate. It should be emphasized that these results are based on the deformation theory and are not generally valid for unloading or cyclic loads.

\section{EXPERIMENTAL PROGRAM}

Because of the complexity and controversy surrounding the issue of interface cracks, an experimental program aiming at establishing empirical failure criteria under combined tensile and shear loading has been first undertaken and is currently in progress. Aluminum/epoxy specimens with dimensions 200X77X5 in mm as shown in Fig. 5(a), have been produced by casting the epoxy in a mold containing the aluminum, which has been cleaned and etched with a sulfuric acid solution to improve the bond. Without appropriate etching the residual stresses produced as a consequence of the curing process are high enough to break the bond. The aluminum 2024-T4 was donated by ALCOA, and the epoxy DER 331 and curing agent DEH 24 were donated by Dow Chemical Corporation. The epoxy was reinforced with four pieces of fiberglass at the location of the bolts to prevent tearing. The interface slit was originally obtained by attaching teflon tape on a centered segment of the interface, and later by simply filing away the surface etching at the location of the intended slit. This procedure assured a very weak bond, which served consistently as the crack initiation site, and eliminated bubbles caused by the air trapped under the teflon tape. The angle of the interface was $25^{\circ}$ from the loading axis, other angles being currently under study. The specimens were cured for two hours at $100^{\circ} \mathrm{C}$ and cooled at room temperature. Constant maximum load fatigue tests were performed to determine the cycles to failure and the modes of fracture. The fracture initiated at the top of the weak bond and propagated along the interface and into the epoxy, as shown in Fig. 5(b). Figure 6 shows the number of cycles to failure versus the standard deviation for three load ratios, $R=0.18,0.055,-0.007$. The maximum applied load was $3.3 \mathrm{kN}$. The angle of fracture in the epoxy varied from $12^{\circ}$ to $30^{\circ}$ from the normal to the interface. The theoretical value computed from the asymptotics, Comninou[11], if we assume that the crack propagates initially in the direction of maximum tensile stress is $16^{\circ}$.

The fracture surfaces, shown schematically in Fig. 7, were studied under the scanning electron microscope (SEM), and pictures were taken at the locations shown. The fracture surface of the epoxy showed three distinct regions: (i) a region next to the interface which had a smooth and shiny appearance, and corresponded to the initial, sudden crack, Fig. 7(e); (ii) a region with radial 


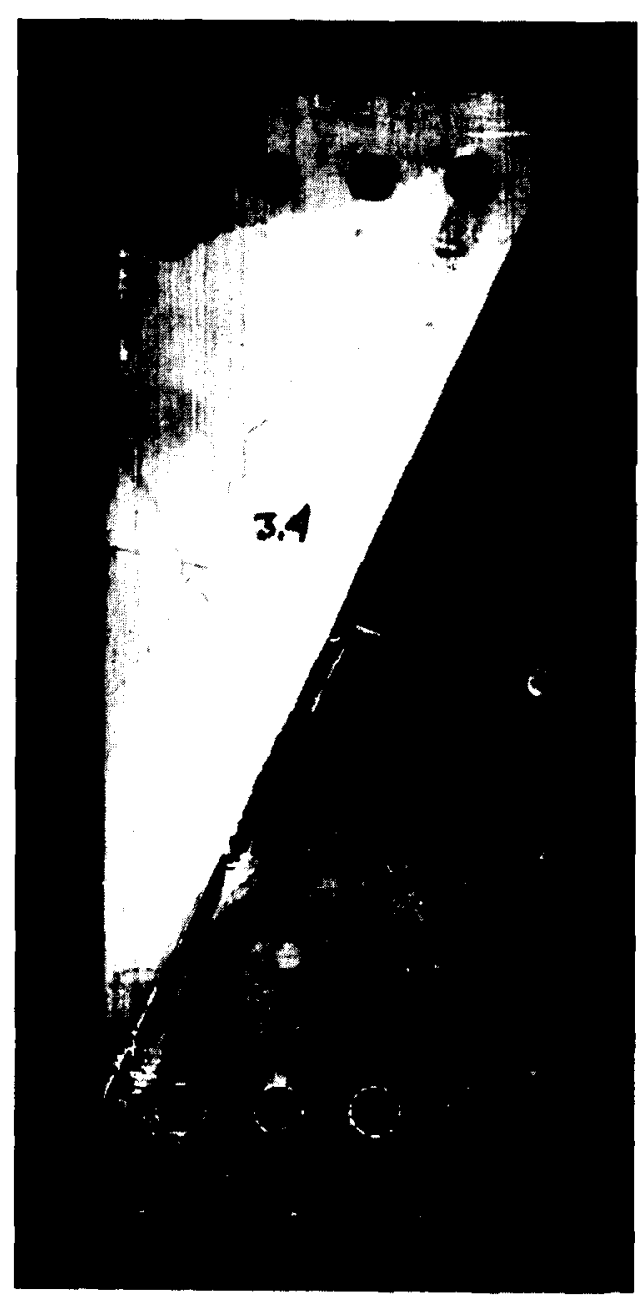

(a)

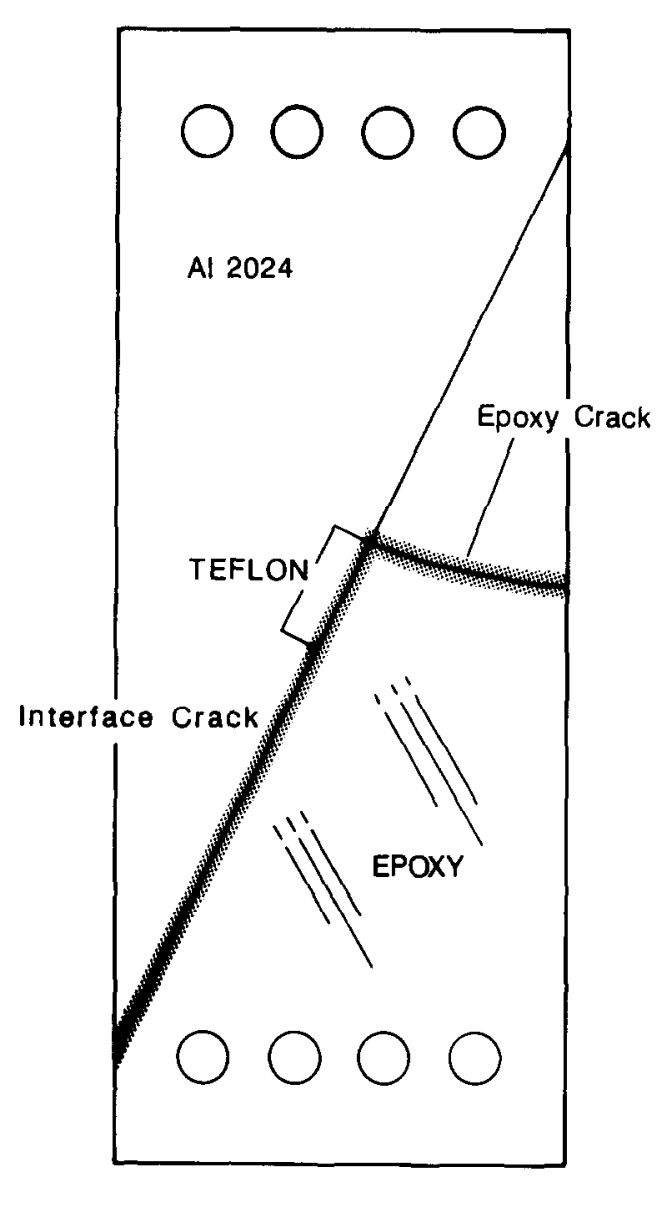

(b)

Fig. 5. (a) Specimen configuration, (b) crack path. 

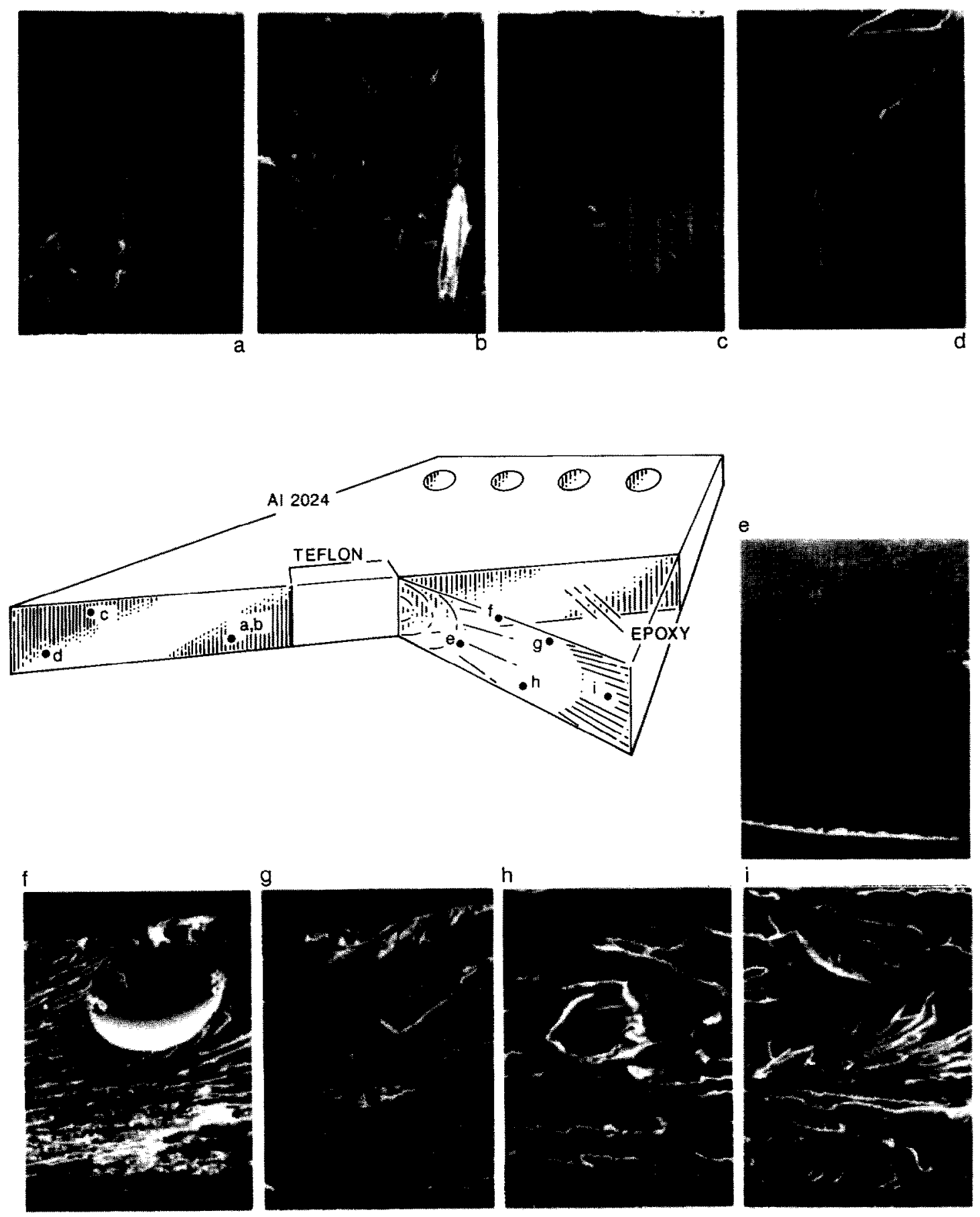

Fig. 7. SEM pictures of the fracture surfaces. 

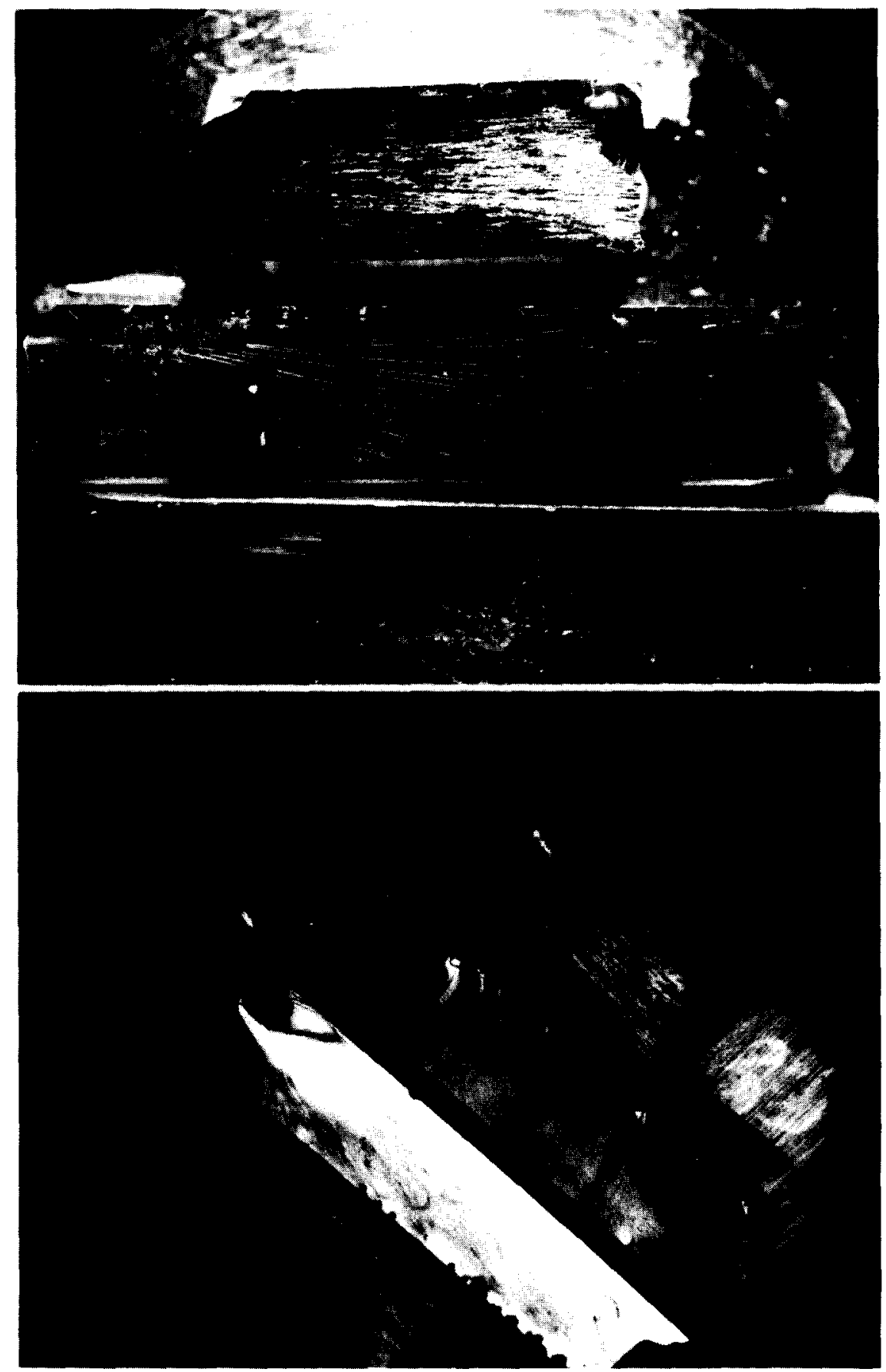

Fig. 8. Representative fracture surfaces of the epoxy crack. 

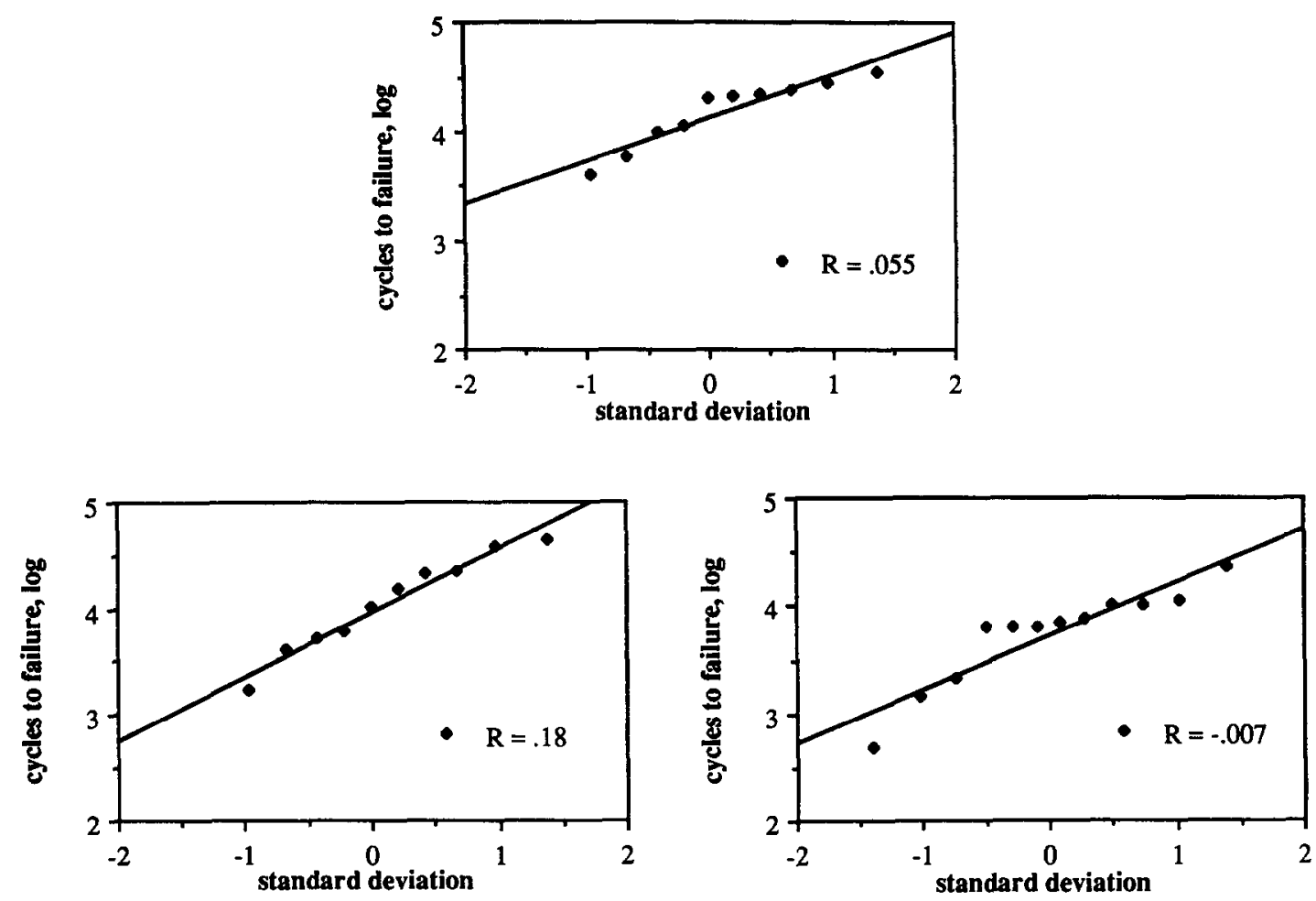

Fig. 6. Cycles to failure vs standard deviation for three $R$ values.

markings emanating from region (i), Fig. 7(f), (g), (h); and (iii) a final region with coarser markings, Fig. 7(i). Crazing or welts are clearly visible in Fig. 7(h). An air bubble is seen in a region of radial markings in Fig. 7(f). One may also observe some unidentified markings resembling tooth marks in Fig. 7(g). The interface crack surface showed strips of epoxy adhering in the aluminum, Fig. 7(a), (c). Ftching pits filled with epoxy can be seen in Fig. 7(d), and a detail of an epoxy strip is shown in Fig. 7(b). Figure 8 shows representative fracture surfaces of the epoxy crack.

In the configuration of the specimens, the interface is subjected to a combined tension and shear, at a ratio of approximately 0.5 . For this value, the extent of the finite contact zone is predicted from the linear analysis to be approximately $10 \%$ of the crack length, Comninou and Schmueser[23]. Such contact zones has been observed experimentally under static loads, Liechti and Knauss[27].

Acknowledgements - The experimental program described in this paper was made possible by a grant of the Rackham School of Graduate Studies of the University of Michigan and by the support of the Mechanical Engineering and Applied Mechanics department. The help and advice of Mr. W. H. Durrant, and the work of graduate student John Sapp and undergraduate student Debborah Billings in the laboratory, are also gratefully acknowledged.

\section{REFERENCES}

[1] M. L. Williams, The stresses around a fault or crack in dissimilar media, Bull. Seismological Soc. America 49, $199-404$ (1959).

[2] F. Erdogan, Stress distribution in a nonhomogeneous elastic plane with cracks, J. Appl. Mech. 30, 232-237 (1963).

[3] J. R. Rice and G. C. Sih, Plane problems of cracks in dissimilar media, J. Appl. Mech. 32, $418-423$ (1965).

[4] A. H. England, A crack between dissimilar media, J. Appl. Mech. 32, 400-402 (1965).

[5] B. M. Malyshev and R. L. Salganik, The strength of adhesive joints using the theory of fracture, Int. J. Fracture Mech. 1, 114-128 (1965).

[6] J. Dundurs, Discussion, J. Appl. Mech. 36, 650-652 (1969).

[7] F. Erdogan and G. D. Gupta, Layered composites with an interface flaw, Int. J. Solids and Structures, 7, 1089-1107 (1971).

[8] J. Rice, Elastic fracture mechanics concepts for interfacial cracks, J. Appl. Mech. 55, 98-103 (1988).

[9] J. W. Hutchinson, M. Mear and J. R. Rice, Crack paralleling the interface between dissimilar materials, J. Appl. Mech. 54, 828-832 (1987). 
[10] C. Atkinson, On stress singularities and interfaces in linear elastic fracture mechanics, Int. J. Fracture 13, 807-820 (1977).

[11] M. Comninou, The interface crack, J. Appl. Mech. 44, 631-636 (1977).

[12] G. Fichera, Boundary value problems of elasticity with unilateral constraints, in Handbuch der Physik, VIa/2, Springer, Berlin (1972).

[13] R. T. Shield, Uniqueness for elastic crack and punch problems, J. Appl. Mech. 49, 516-518 (1982).

[14] H. D. van der Zande and H. J. Grootenboer, A finite element approach to interface cracks, J. Appl. Mech. 53, 573-578 (1986).

[15] C. Atkinson, The interface crack with a contact zone, Int. J. Fracture 18, 161-177 (1982).

[16] A. K. Gautesen and J. Dundurs, The interface crack in a tension field, J. Appl. Mech. 54, 93-98 (1987).

[17] A. K. Gautesen and J. Dundurs, The interface crack under combined loading, J. Appl. Mech. (1988). (in press).

[18] S. Itou, Stresses around an interface crack, Engng Fracture Mech., 25, 415-420 (1986).

[19] X. S. Zhang, A central crack at the interface between two different media for the opening mode, Engng Fracture Mech. 24, 177-185 (1986).

[20] J. H. Park and Y. Y. Larmme, Application of conservation integrals to interfacial crack problems, Mech. Mater. 5, 261-276 (1986).

[21] M. Comninou, The interface crack in a shear field, J. Appl. Mech. 45, 287-290 (1978).

[22] M. Comninou and J. Dundurs, On the behavior of interface cracks, Res Mechanica 1, $249-264$ (1980).

[23] M. Comninou and D. Schmueser, The interface crack in a combined tension-compression and shear field, J. Appl. Mech. 46, 345-348 (1979).

[24] J. K. Knowles and E. Sternberg, Large deformations near a tip of an interface crack between two neo-Hookean sheets, J. Elasticity 13, 257-293 (1983).

[25] J. K. Knowles, A nonlinear effect in mode II crack problems, Engng Fracture Mech. 15, 469-476 (1981).

[26] C. F. Shih and R. J. Asaro, Elastic-plastic analysis of cracks on bimaterial interfaces. Part I: small scale yielding, $J$. Appl. Mech. 55, 299-316 (1988).

[27] K. M. Liechti and W. G. Knauss, The use of crack profile measurements to determine mode interactions of propagating cracks of material interfaces, in 1981 Advances in Aerospace Structures and Materials (Edited by S. S. Wang and W. J. Renton), ASME, New York (1981). 